Murmurations:

Journal of

Transformative

Systemic

Practice
READING NOTES

\section{Aspects of Reading}

\section{Desa Markovic}

Volume 3

Issue 2

Spring 2021

Keywords:

reading,

reflexivity,

critique

\section{Citation Link}

\section{Abstract}

As a tutor and a teacher on various psychotherapy and supervision courses over time, I became aware of the need for a structured framework that would guide discussions on the literature during the sessions with students.

The feedback from tutors and students has encouraged me to continue to use it and particularly emphasised its potential to stimulate self-reflexive thinking.

The Model is presented as a format for reading papers, discussing the material with others, and giving an account of reading such as writing a critique or a literature review. The purpose of the 'Aspects of Reading' is to enhance possibilities for learning by highlighting different positions a reader can take during these activities. 


\section{Aspects of Reading}

\section{Limitations}

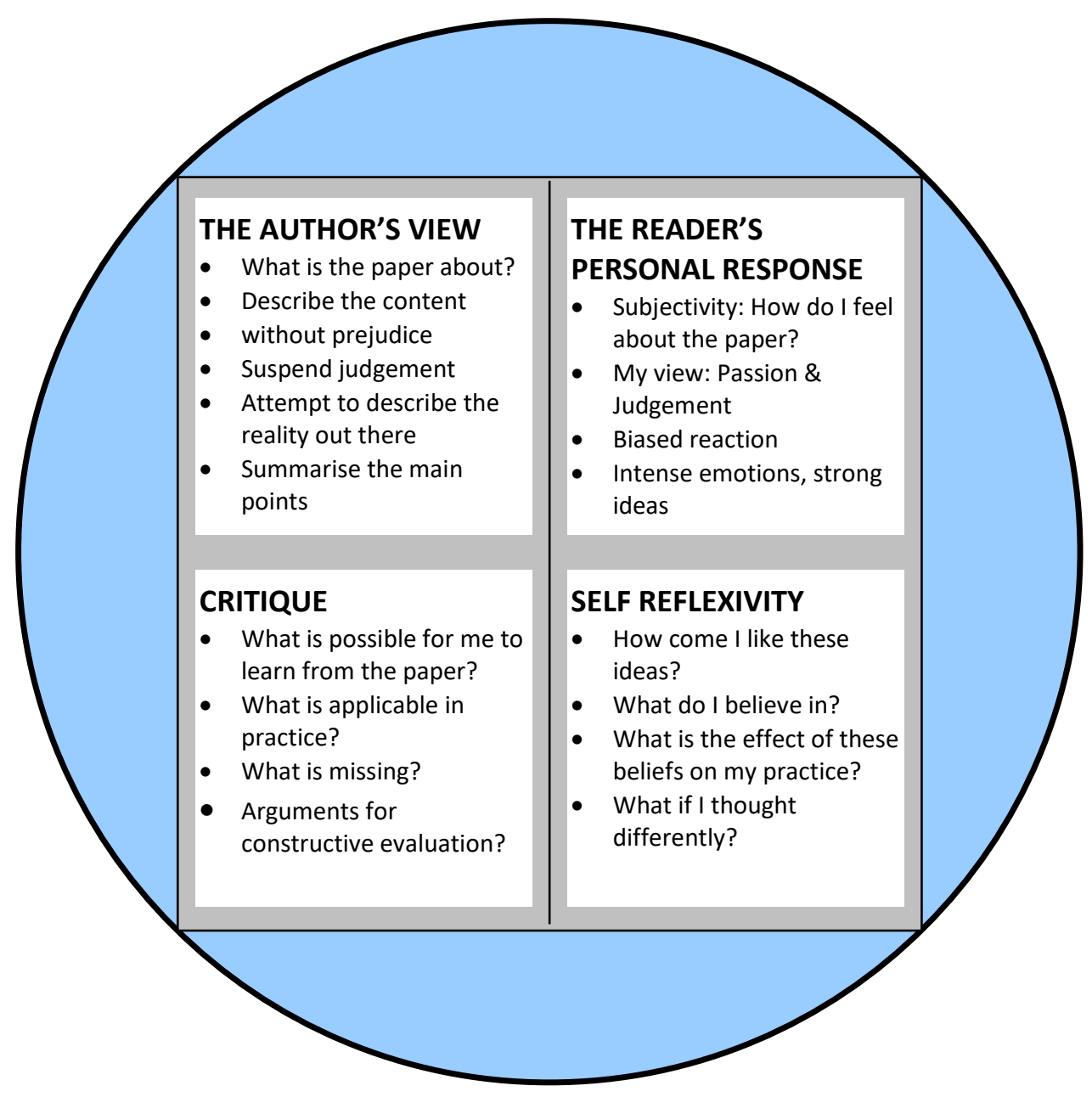

Multiple Context Influences 


\section{The Author's View}

\section{THE AUTHOR'S VIEW}

\section{Summary:}

- An ability to give a clear outline of the main points from the content

- Being disciplined and attentive to 'factual information'

- Describing the 'reality out there'

- Taking an objective position

Guideline specific questions:

- What is the paper about?

- What do the main ideas and concepts refer to?

- What are the main arguments about?

- When was it written?

- What is the author's theoretical/ epistemological / ethical position?

- What is the proportion of theory in relation to practice?

This aspect of the Model could be alternatively named: 'The Position of Objectivity'; 'Non-Neutrality', or: 'The Summary of the Paper's Content'. From this position, readers are encouraged to give a concise overview of a paper whilst suspending their views on it.

In certain contexts, it can be helpful to provide a clear summary of the main points from the paper's content, for example when doing a literature review on a particular topic or when participating in a discussion on the subject that a paper addresses.

The act of reading is often mixed with personal responses to the written material which can interfere with the memory of the paper content. The discipline of adopting this particular aspect of reading, i.e. 'The Author's View', and the focus of attention being put on the 'what' questions that address specific content details and other relevant - as factual as possible - information, ensures a better recollection of the paper and less of a mix between 'What do I feel about the paper?' and 'What is the paper about?'

Whilst being aware that stating objectivity can be seen as inherently pre-modern, I believe that careful, systematic, and thoughtful readers should be able to demonstrate their clear knowledge of a piece of a literature. For example, they should be able to answer the questions such as:

- What is the paper about?

- How long is it?

- When was it written?

- What are the main points, ideas and concepts referred to?

This position is to do with deliberately trying to be 'non - systemic' and for a limited period of time believing that there are such things as 'facts', 'hard reality' and a world as it happens 'out there'. At the same time, when acting from this position, a systemic thinker would be aware that there may be 
numerous ways in which a paper could be summarised and different views on what might constitute the 'main ideas'.

When applying this Model during their course, students repeatedly commented on the value of 'The Author's View', recognising how often they came across a critique of papers based on third party critique rather than on the original source. They also reflected how easy it is for them to fall into the trap of basing their ideas on other people's critique when not taking this rigorous position. This also draws attention to the significance of putting the original texts on the reading lists of family therapy courses.

\section{The Reader's Personal Response}

\section{THE READER'S PERSONAL RESPONSE}

Summary:

- A biased, emotional, subjective view of the reader

- Includes criticism, praise or indifference

- Trainers can helpfully encourage expression of trainees' personal reaction

- Going beyond being right, clever, or systemic

Guideline specific questions:

- How do I feel about the paper?

- If it was sitting here, what would I say to it?

- What opening statement could I write about it?

- What do I think should happen to the paper?

- How should it be celebrated?

- How should it be destroyed?

The alternative names for this aspect could be: 'The Position of Subjectivity'; 'Passion and Judgement' or 'The Reader's View'. From this position, the reader allows all the judgement and emotions previously suspended to be expressed as intensely as they were felt during the reading, for example: 'What a difficult paper'; 'Too long'; 'Well written'; 'Uninspiring', and so on. Comments like these are the reader's personal reaction and are neither an objective, factual, descriptive account, nor are they a constructive, evaluative account of the paper. These are biased, judgemental, or sometimes emotional views of the readers, to which they are entitled whether they are able to justify them or not.

Validating the Personal View Position allows for a greater sense of freedom to openly express personal opinions without taking them too seriously, or worrying about being right. Connecting with emotions and naming them is important within the Model so that the Personal Position is clearly distinct both from a descriptive account ('The Author's View') and from a critique of the paper. 
Listening to personal responses to reading, including indifference, and describing it to others can be a useful exercise and beneficial for readers in terms of learning about their own biases, prejudices and resources, thus informing the process of self reflection.

This is an aspect of reading that is not for debate; it is more of a sharing discussion that encourages expression. Sometimes it works well if done before moving on to the critique as it emphasises the difference between the personal response and the critique where a debate usually takes place.

\section{Critique}

\section{CRITIQUE}

Summary:

- A constructive approach taken with a viable rationale

- An evaluative attitude to giving a view on the paper

Guideline specific questions:

- What is the paper's contribution?

- How does it enrich my understanding of the topic and / or of the systemic approach?

- What ideas from the paper could be most usefully applied to practice?

- What are the paper's limitations?

- What is missing?

- How could it be improved?

- What are the useful learning points?

- What is the author's ethical position, for example how sensitive is the paper to power differential?

- What ideas are privileged?

Critique is often usefully enlightened by the strong ideas and emotions from the Personal Position so it can appear passionate and intense. This intensity is of a different quality to the Personal Position as it is supported by 'The Author's View'. The two positions combined can produce a constructive, well documented critique.

An evaluative, constructive approach to giving a view on a paper relates to questions such as:

- What is the paper's contribution?

- What are its shortcomings?

- What ideas could be most usefully applied to practice?

- What is missing?

A useful exercise in reflexive reading would be to invite students to give an affirmative account of a paper they feel negative about; and vice versa, to provide a critique on the shortcomings of a paper that has triggered positive personal responses. 


\section{Self Reflexivity}

\section{SELF REFLEXIVITY}

Summary:

- Exploring the reader's relationship with the paper

- Examining readers' values, choice, and the effects of their beliefs on their practice

Guideline specific questions:

- How come I have this view?

- What position am I privileging?

- What could it be that is stopping me from having a different view?

- What do I think of others who think differently to me?

- How do the key ideas fit with my preferred theoretical frame?

- How do these ideas fit my personal style?

- What is the impact of me reading this paper at this point in time in my professional development?

- What effect will it have on my future development and interests such as supervision, research, etc.?

- Which of my cherished ideas / beliefs do these ideas challenge?

- What might I have to give up in order to incorporate them in my work?

- What would be the other challenges for me in using these ideas in my clinical practice?

- Who would be the first to know of these changes?

- How would they be affected?

- How does reading this paper affect my view of myself as a systemic therapist?

- How might it be different if I was male / female?

- What difference would it have made to my professional development if I had read this paper five years ago?

- How do these ideas affect my view of the world?

- What part do these ideas have in my personal development?

This aspect is about the readers' beliefs and their relationship with the paper, and how it connects with their professional self image. It relates to curiosity regarding the self of the readers, their own values, choices, and the effects of those on their practice. A self reflexive position can enhance the position of critique in that it encourages questioning the readers' questions and thus re-examining their beliefs and opinions.

The following questions can guide curiosity in this position: How come I have this view? What position am I privileging? What could it be that is stopping me from having a different view? What do I think of others who think differently to me? How do the key ideas fit with my preferred theoretical frame? What is the impact of me reading this paper at this point in time in my professional development? What effect will it have on my future development and interests such as supervision, research? 


\section{Limitations / Multiple Context Influences}

\section{LIMITATIONS / MULTIPLE CONTEXT INFLUENCES}

Summary:

- Limits are inevitable context markers

- Considering the purpose of the paper

- Taking into account the contexts (cultural, historical, philosophical, ethical, theoretical) of the paper and of the reader

Guideline questions:

- What is the historical and cultural context in which the paper was created and to which it relates?

- What is the author's cultural (gender, sexuality, race, culture, ethnicity, class) background?

- What is the reader's cultural (gender, sexuality, race, culture, ethnicity, class) background?

- How could considering the interplay of the above enrich the critique?

The four positions on the diagram are surrounded with the space represented by a circle, indicating that the positions are inevitably limited however they are used or formulated, including recognition of the limitations of the language itself. The circle is there as a reminder that an awareness of the kinds of limitations in a particular paper can enhance readers' understanding of it. For constructive and self reflexive reading, it is important to define limitations in reading and describing a paper. For example, the parameters in reading some papers might be: 'It is only one chapter in a book that I have not read'; 'My knowledge of the topic is limited'; 'The paper is breaking new ground, stepping into a new territory with not much prior knowledge to support it', as some students have thoughtfully remarked in the past.

Limitations also refer to the multiple context influences to be considered when trying to understand, evaluate and learn from the reading and might include context markers such as: philosophical, ethical and theoretical framework, worldview, social norms and approved practices, and prevailing value systems of the authors and readers. A constructive critique would consider the purpose of a paper and evaluate it in ways that would be context relevant, respecting its limits as inevitable context markers, determining the paper's boundaries.

Some guideline questions, appreciating the paper's limitations, could be:

- What is the historical and cultural context in which the paper was created and to which it relates?

- What is the author's cultural (gender, sexuality, race, culture, ethnicity, class) background?

- What is the reader's cultural (gender, sexuality, race, culture, ethnicity, class) background?

- How could considering the interplay of the above enrich the critique? 


\section{Applicability of the Model / Concluding Comments}

This Model has been developed over time and in response to my experience of trainees' need of enhancing evaluative and self reflexive skills when reading and discussing papers or reviewing tapes of their clinical work. While it may not be totally comprehensive, it highlights different aspects of the process and of the outcome of reading. The structure of this Model allows for four clearly defined positions, and gives validity to each by emphasising the distinctions between them.

At certain times different positions can be more or less relevant and at others it can be helpful to be able to decide to deliberately speak from a particular position. This Model can be also used as a tool for developing self reflexivity, for example observing patterns over time and reflecting which positions the readers take more / less frequently and to question for what reasons.

The use of the Model is more active and the four positions are more connected, informing and influencing each other, than appears from a static diagram. In one sentence it is possible to move between positions and connect them, for example: 'When I feel this way, what does it allow me to learn from this particular paper?' Trainers on family therapy courses can enable students to enhance their critical literary skills by using questions that would help them make connections and distinctions between different aspects of reading.

\section{Acknowledgement}

I wish to thank Teresa Wilson for her helpful reading of the final draft.

\section{Author}

Desa Markovic, PhD, is a Professor in Systemic Psychotherapy, a UKCP accredited systemic psychotherapist and a COSRT accredited psychosexual therapist and supervisor. She has written extensively on the topic of integration between systemic psychotherapy and sexology.

E-mail: desamarkovic@btinternet.com

URL: http://www.coventgardencounselling.com/

\section{Citation}

Markovic, Desa (2021). Aspects of Reading. Murmurations: Journal of Transformative Systemic Practice, 3, 2, 129-136. https://doi.org/10.28963/3.2.10 